\title{
APPLICATIONS OF THE ARGUS CODE IN ACCELERATOR PHYSICS*
}

J.J. Petillo, A. Mankofsky, W.A. Knueger, C. Kostas, A.A. Mondelli, and A.T. Drobot Science Applications Intermational Corporation, McLean, VA 22102

\section{ABSTRACT}

$$
\text { CONF-9302104--3 }
$$

ARGUS is a three-dimensional, electromagnetic, particle-in-cell (PIC) simulation code that is being distributed $10 \mathrm{U} . \mathrm{S}$. accelerator laboratories in collaboration between Science Applications International Corporation (SAICTM) and the Los Alamos Accelerator Code Group (LAACG). It uses a modular architecture that allows multiple physics modules to share common utilities for grid and structure input. memory management, disk VO, and diagnostics. Physics modules are in place for electrostatic and electromagnetic field solutions, frequency-domain (eigenvalue) solutions. time-dependent PIC, and sieady-state PIC simulations. All of the modules are implemented with a domain-decomposition architecture that allows large problems to be broken up into pieces that fit in core and that facilitates the adaptation of ARGUS for parallel processing. ARGUS operates on either Cray or workstation platforms, and a MOTIF-based user interface is available for $X$-windows terminals. Applications of ARGUS in accelerator physicas and design are described in this paper.

\section{DESCRIPTION OF ARGUS}

\section{MASTER}

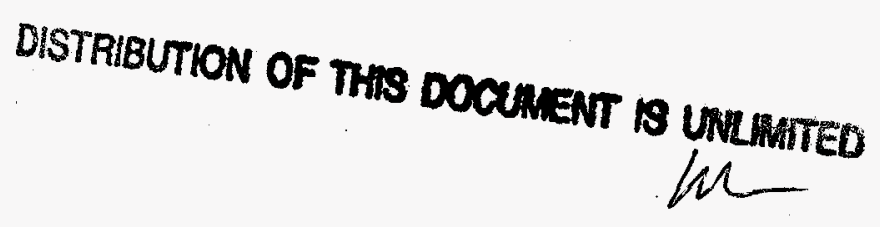

The ARGUS codel,2 has been under development at SAIC since 1983. It is a general-purpose three-dimensional simulation code. The code architecture is specifically designed to handle the problems associated with three-dimensional simulations. It uses sophisticated memory management and data handling iechniques $^{3}$ to deal with the large volume of data that is generated in three-dimensional simulations. Recent work by Leabrook Computing. Lid. shows that the domain decomposition technique used in AROUS provides a suitable framework for coarsegrained parallelization of the code.

A modular architecture is employed so that ARGUS is in fact a system of threedimensional codes (numerical modules) that utilize a common data structure and share utilities for structure input, grid generation, memory management, data handling, and diagnostics. The codes allow complicated geometrical structures to be represented on the computational grid. The grid can be nonuniform in all three dimensions. Cartesian and cylindrical coordinate systems have been implemented throughout AROUS; some of the modules also support toroidal and mixed coordinates. Physics modules are in place to compute electrostatic and electromagnetic fields, the eigenmodes of if structures, and PIC simulation in either a timedependent mode or a steady-stane mode. The PIC modules include multiple particle species, relativistic particle dynamics, and algorithms for the creation of particles by emission from material surfaces and by injection onto the grid. A plasma chemistry module allows species to be creared or destroyed based on specified rate processes.

The structure input in ARGUS is carried out through combinatorial geometry. The code stores a library of basic three-dimensional objects (c. g., a rectangular solid, an elliptical cylinder, an ellipsoid, etc.). These objects are combined by the user with logical operations (to either add or delete the library object) to produce structures of antitrary shape. The siructures so specified are represented on the computational grid 


\section{DISCLAIMER}

This report was prepared as an account of work sponsored by an agency of the United States Government. Neither the United States Government nor any agency thereof, nor any of their employees, makes any warranty, express or implied, or assumes any legal liability or responsibility for the accuracy, completeness, or usefulness of any information, apparatus, product, or process disclosed, or represents that its use would not infringe privately owned rights. Reference herein to any specific commercial product, process, or service by trade name, trademark, manufacturer, or otherwise does not necessarily constitute or imply its endorsement, recommendation, or favoring by the United States Government or any agency thereof. The views and opinions of authors expressed herein do not necessarily state or reflect those of the United States Government or any agency thereof. 


\section{DISCLAIMER}

Portions of this document may be illegible in electronic image products. Images are produced from the best available original document. 
by a structure mask array, which stores the material and electrical properties of each cell on the grid

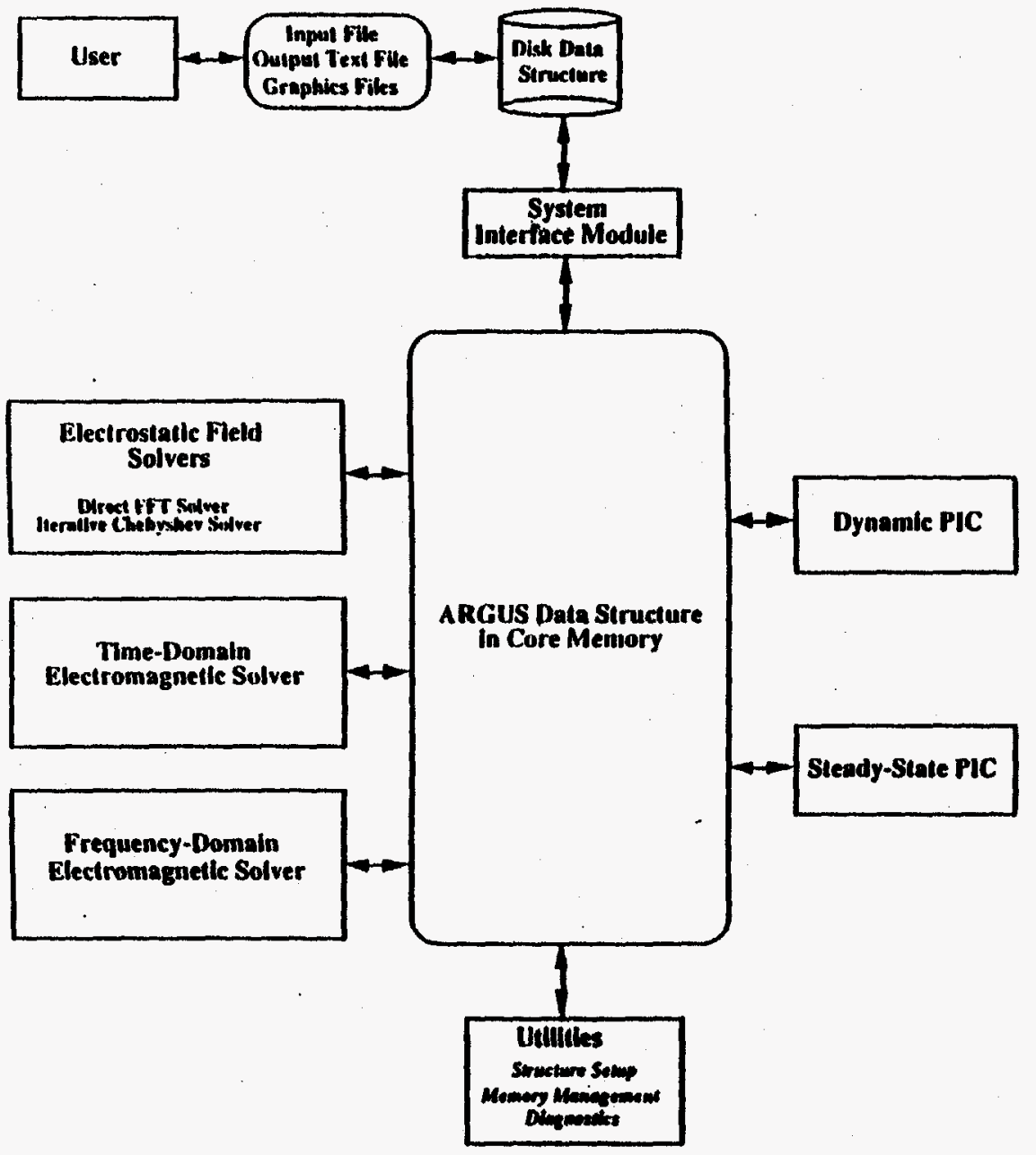

Figure 1. ARGUS modular code arebllecture.

Material properties can be associated with structures. The code allows perfectly-conduciong materials, as well as materials with complex values of both permittivity and permeability; hence lossy materials are allowed. Furthermore, the permittivity and permeability may be specified as diagonal tensors to treat certain classes of non-isotropic materials.

ARGUS has a wide variety of diagnostic plots that are selected by user input and are available at run time. These include set-up graphics, contour and arrow plots of field quantities, and plots of particle trajectories and phase space. The code also will allow the user to create HDF files for exporting data to other visualization tools.

\section{SOME EXAMPLES FROM TIE ARGUS PRIMER}

The current code release (ARGUS v.25) includes an ARGUS Primer, which provides a tutorial for new. ARGUS users. It presents a set of simple example problems that illustrate how to use the features in ARGUS. The Primer will also be used in testing future ARGUS releases and ARGUS installations on new computing platforms. A subset of the examples featured in the Primer are presented here.

\section{The "Tombstone" Cavity}

An example in the MAFIA User's Guide consists of analyzing the normal modes of the siructure shown in Figure 2. The structure is a cylindrical drift tube altached 10 a cavity made of a rectangular box topped with a half cylinder. MAFIA and ARGUS represent this structure in different ways. ARGUS uses a "stairstep" representation on the grid, while MAFIA allows triangular "half cells". (Half-cell inasking will be included in a future ARGUS release.)

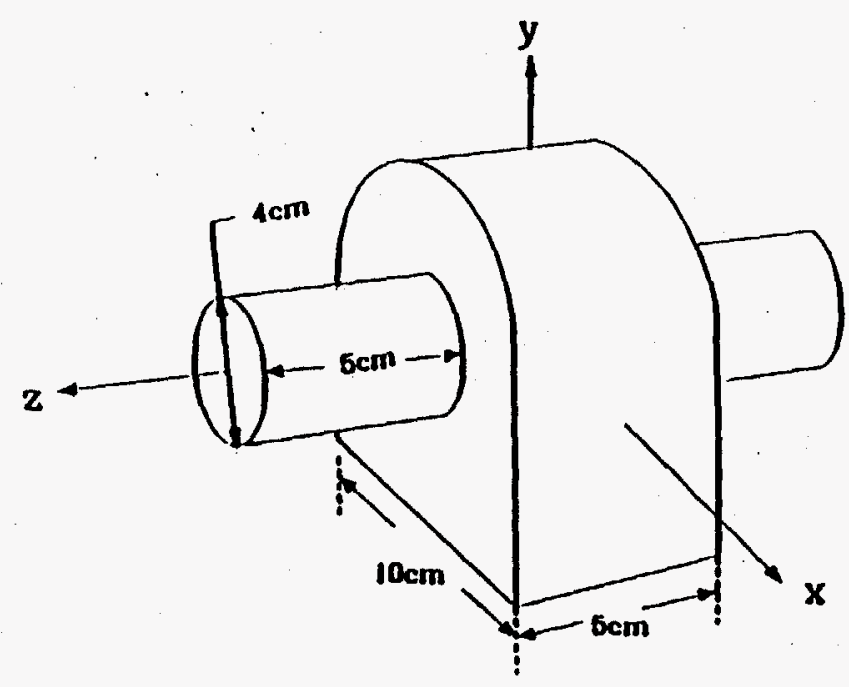

Trure 2. The "Tombstone" carliy.

\section{Cold Test of the "Tombstone" Cavity}

A comparison of ARGUS and MAFIA cold-test results for the "Tombstone" cavity are given in Figure 3. The ten lowest modes of the stmucture were computed. The discrepancy is generally in the 1-2\% range, and is due to the differences in the structure representation by the two codes. This result is consistent with other comparisons of these codes on comparable meshes. With finer meshing, the codes agree more closely. 


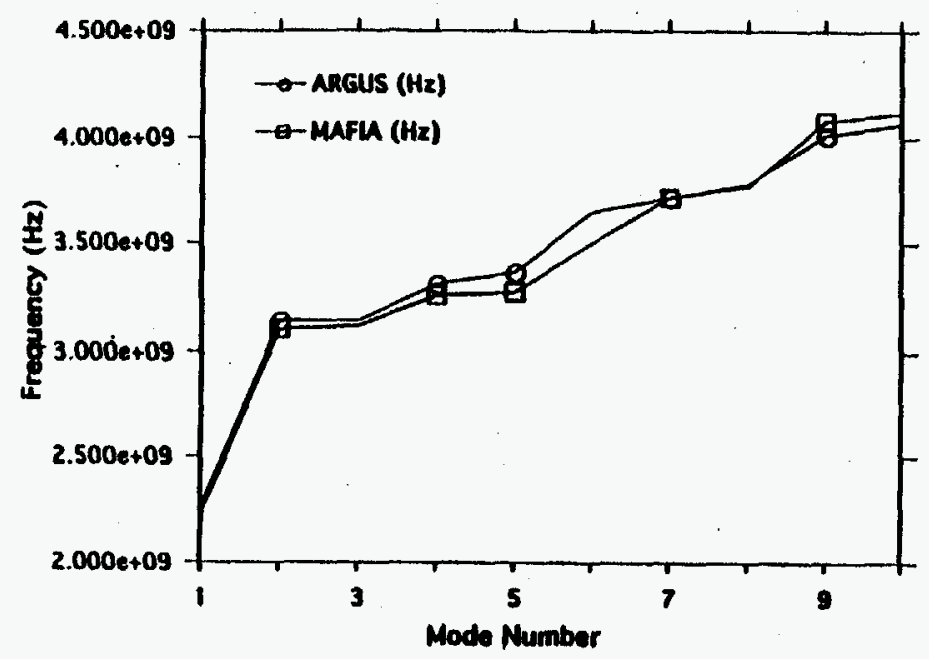

Fileure 3. Comparison of ARGUS and MAFIA resullus for "Tomberome" cavily cold teat.

Wake Fields in the "Tombstone" Cavity

Inne slep 25

eleclicic lield (Ex) - lime elep. 75

live slep 125
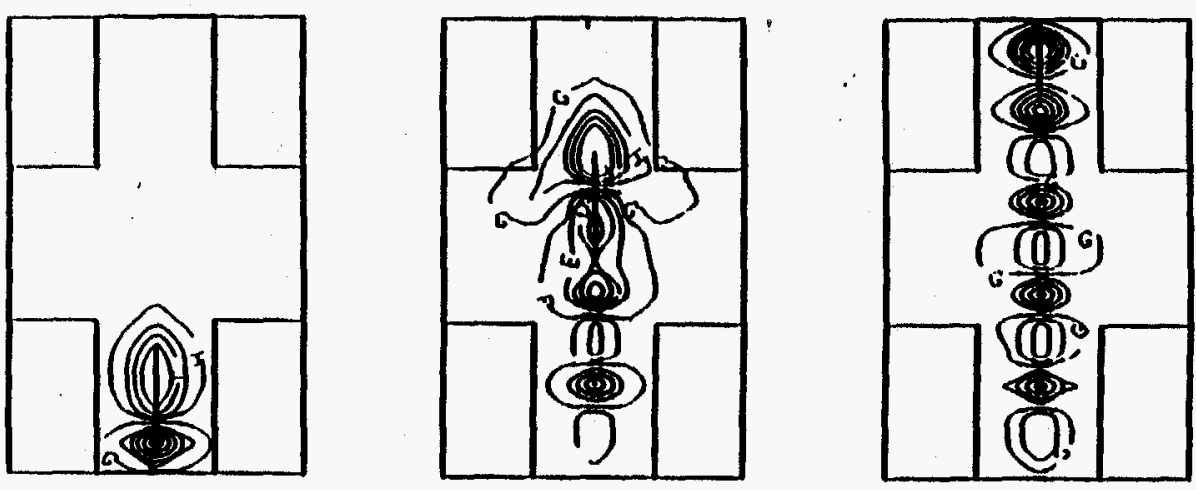

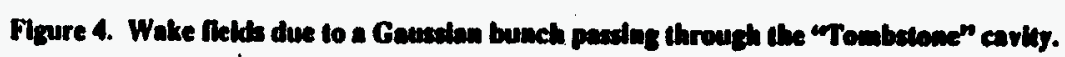

By initializing a Gaussian bunch of heavy particles in the time-dependent PIC module, and allowing them to drift through a stnucture, the user can cause ARGUS to create wake fields in the structure. This method was employed to display the wake fields in the "Tombstone" cavity. Figure 4 shows three snapshots of the particles and axial electric field as the bunch traverses the structure. The Gaussian bunch was placed on the axis of the cylindrical driff tube and launched into the tombstone cavity The particles are visible in the figure as line charge on axis. In the first frame. time step 25, the particles are just entering the tombstone cavity from the lower drift lube. In the second frame, time step 75, they are just leaving the tombstone cavity and entering the upper drifi tube. In the third frame, time step 125, they are leaving the upper drifit tube (by being absorbed on the upper boundary of the simulation).

\section{Radiating Open Waveguide in the Time Domain}

The ability of the ARGUS code to handle open, radiating boundary conditions in the time domain sets it apart from several other codes. Two separate utilities exis for carrying out this capability. The first is a "port" boundary condition, which ineats a specified opening in the simulation boundary as though it were connected to waveguide extending to infinity. The second is an implementation of the Lindman algorithm for radiating boundaries. While the port condition only matches the boundary for radiation at a specified frequency, the Lindman condition is a genera outgoing wave boundary condition.

This simulation provides a demonstration of the Lindman algorithm for the simple example of a rectangular waveguide that is driven at the lower end, and open at the upper end. Figure $\mathbf{5}$ shows the radiation fields at two time steps, selected to show the phase slippage at the top (open) boundary.
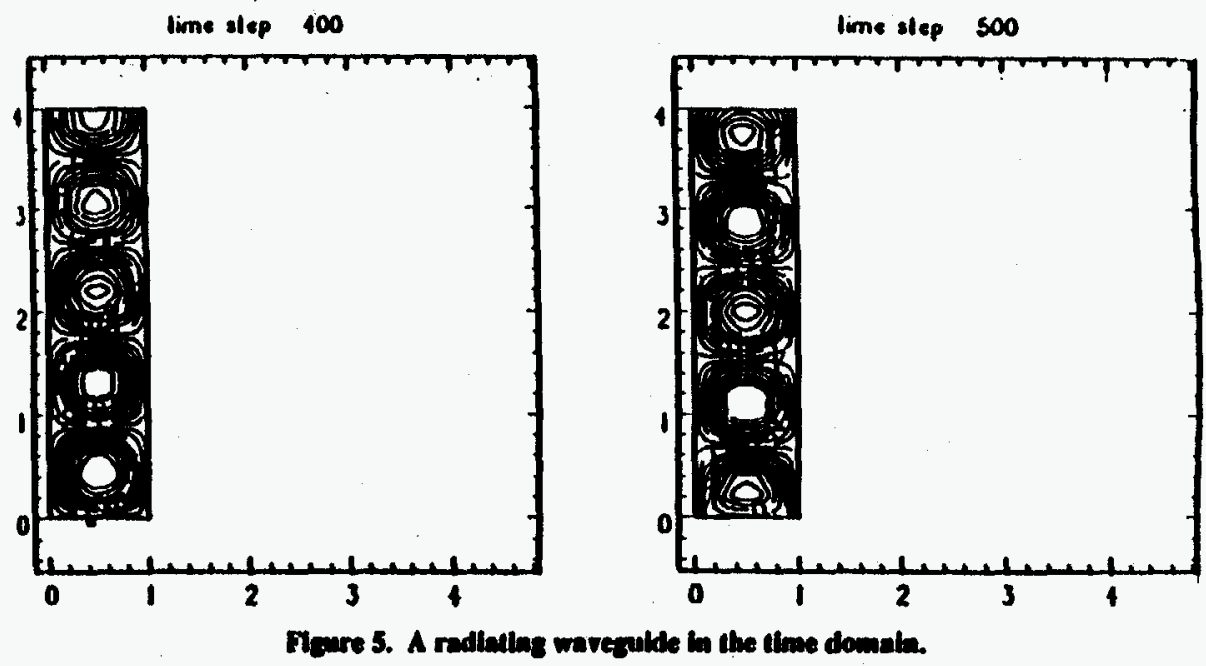

\section{WA VEGUIDE-CAVITY COUPLING}

An important problem in the design of if linacs is the coupling between the waveguide that feeds if power to the accelerator and the cavity through which the beam is accelerated. The designer needs to know the coupling coefficient, the frequency shift. and the external $Q$ due to the waveguide, as well as the fields in the 
aperture of the coupling iris. This problem is difficult for time-domain simulation codes because accelerator cavities often are very high- $Q$ structures, and therefore require very many rf cycles to fill.

ARGUS has been employed in a collaboration between SAIC and AccSys Technology. Inc. 10 model the external $Q$ of the drifi-tube linac (DTL) cavities in the injector for the Superconducting Super-Collider (SSC). The intrinsic Q of the DTL cavities is approximately 40,000. Figure 6 shows the aperture in the waveguide cavity system, as represented in the ARGUS model. The drifi-lube structures are represented by an "equivalent load," consisting of a dielectric rod.
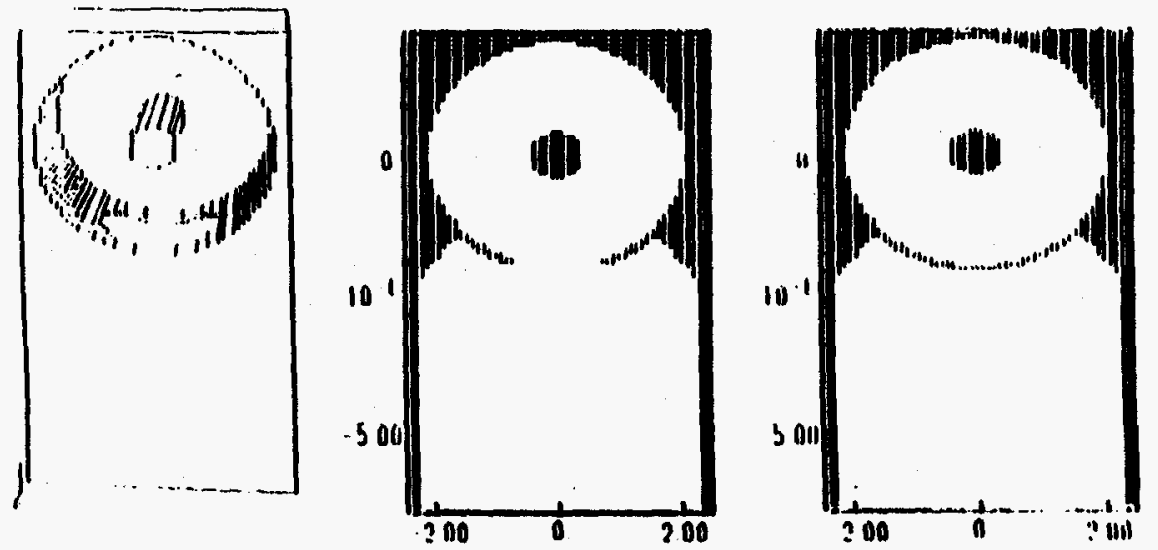

Figure 6. ARGUS representation of the DTL cavily/mavezulde structure for the SSC injector.

The methods used for the solution are based on published techniques $5,6,7$ for using electromagnetic frequency-domain algorithms 10 compute the extermal $Q$ in high- $Q$ cavity-waveguide systems. They require the eigenvalue solver to be exercised several times, with the waveguide shorted al different distances from the iris, thereby allowing a resonance curve of frequency vs. phase shift. like that shown in Figure 7 , to be mapped out. The figure shows the resonance curve for a round iris, $12 \mathrm{~cm}$ in diameter. This analysis is repeated for different choices of the iris dimensions until a suitable design point is realized. The design is a trade-off between the extermal $\mathbf{Q}$, the field strength in the iris aperture, and the cavity mode distortion duc to the aperture.

AccSys has built an experimental test-stand which allowed the ARCUS results 10 be compared with measured data. The results of that test are shown in Figure 8, which presents the external $\mathbf{Q}$ and frequency shift as functions of the iris aperture length, for a fixed aperture width of $12 \mathrm{~cm}$. The experimental data are plotted on the figure and agree with the simulations to within the experimental uncertainty.

AccSys Technology, Inc. is under contract to build the DTL cavities for the SSC Laboratory, and has used these simulations to verify the accuracy of their experimental test data prior to constructing full-scale cavities (which are approximately 10 ill in leng(h). Dr. Jim Potter of AccSys has collaborated with SAIC in this effort. and provided the experimental data which was compared with the ARGUS simulations in Figure 8 .

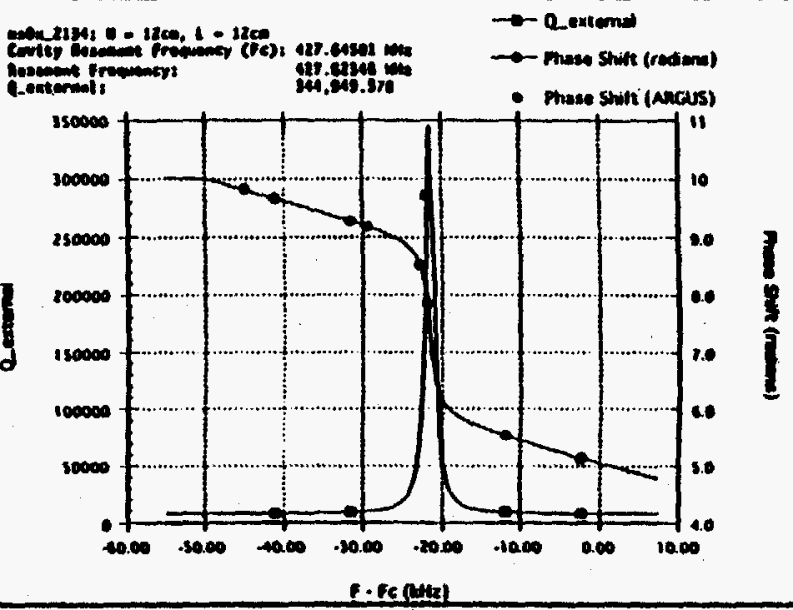

Flgure 7. Phase shill ve. frequency for a $12 \mathrm{~cm}$ dlameter irk aperture.

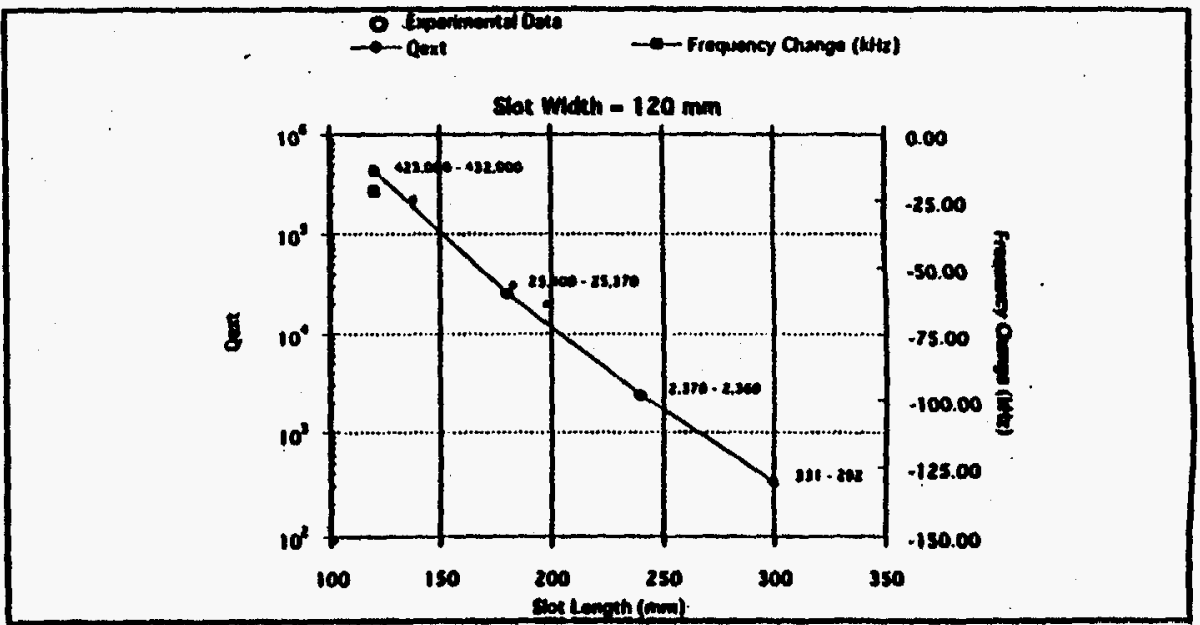

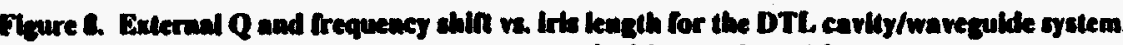
ARGUS stanulation resulls are compared with experimental messurements (courtery of J. Poller, AccSys Technology, Inc.)

\section{HELIX TRA VELING-WAVE TUBE}

The frequency-domain solver in ARQUS v.25 can treat complex fields, where the imaginary part allows the model to treat lossy materials and to handle periodic boundary conditions with sub-phase specification. This latter feature is particularly 
useful when attempting to compute the dispersion curves for long, periodic structures. The usual periodic boundary condition imposes a phase shiff of $2 x$ across the computational grid. If the user needs to impose different phase shin acrose symmetry period of the sfructure, he does so by modeling severn structure periods build up a phase shiti of $2 \pi$ (or $\pi$ with an electric wall symgeveral structure periods to $v .25$ algorthm allows the user to specify the whase shitt to pelane). The ARGUS $v .25$ algorthm allows the user to specify the phase shift to be imposed across the computational grid. thereby enabling him to map out a dispersion curve while modeling only a single structure period.

This module has been used 10 cold lest a helix traveling-wave tube (TWT) developed by Northrop Corporation. Fizure 9 shows the ARCUS representation of the hellx TWT, including the metallic vanes that are used to short the rields between the helix supports. The supports (not shown in the figure) are dielectric rods that attached to the helix and to the outer cylindrical wall. The ARGUS simulation model employed a $80 \times 80 \times 20$ grid, and requested six eigenvectors for each phase shift selected.

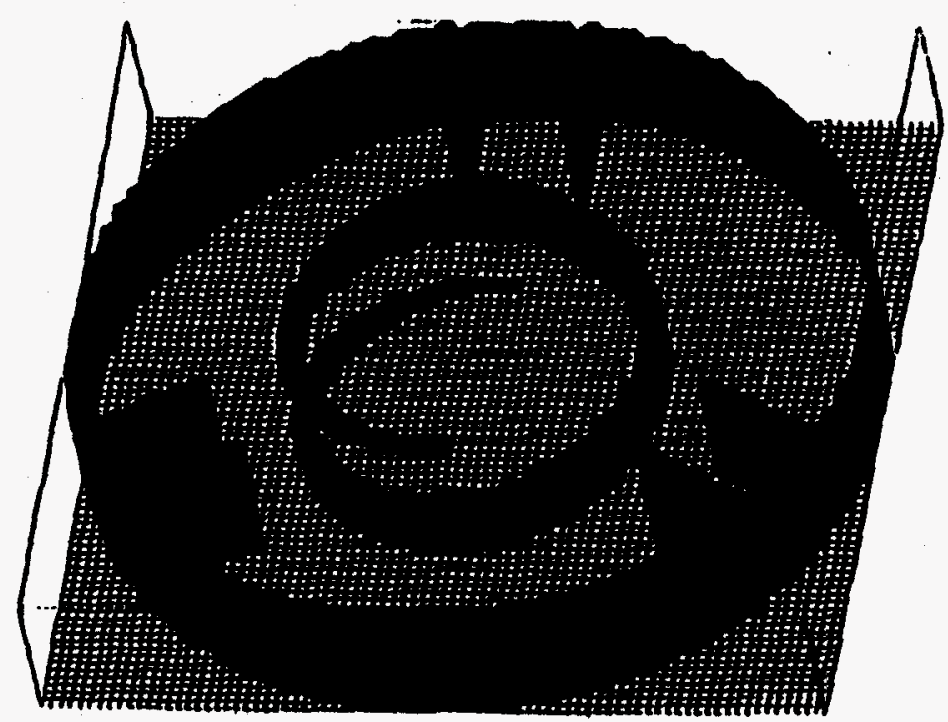

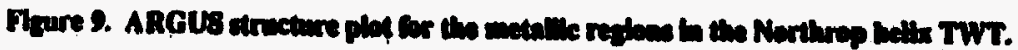

The dispersion curve for the hellx TWT is displayed in Figure 10. The figure shows the effect of the supports and the metallic vanes on the dispersion curve. The vanes are needed to flatien the dispersion curve (l.e., to reduce the dependence of the phase velocity on frequency). They are modeled in ARGUS asence metallic sheets. Most three-dimensional electromagnetic codes are unable to include sub-cell features of this type, and therefore do not succeed in cold test annlyses of devices like the helix TWT. Comparison with experimental data at Nonthrup Indicate that the phase velocity corresponding to Figure lo differs from the measured phace velocity by approximaiely $10 \%$, but this difference can be largely accounted for by adjusting the contact area between the support rods and the helix. (The phase velocity of a helix TWT is known to be very sensitive to the area of contact.)

Dr. Ounther Dochler of Northrup Corporation has graciously provided a description of the helix TWT geometry, and has compared the ARGUS simulation results with his experimental data.

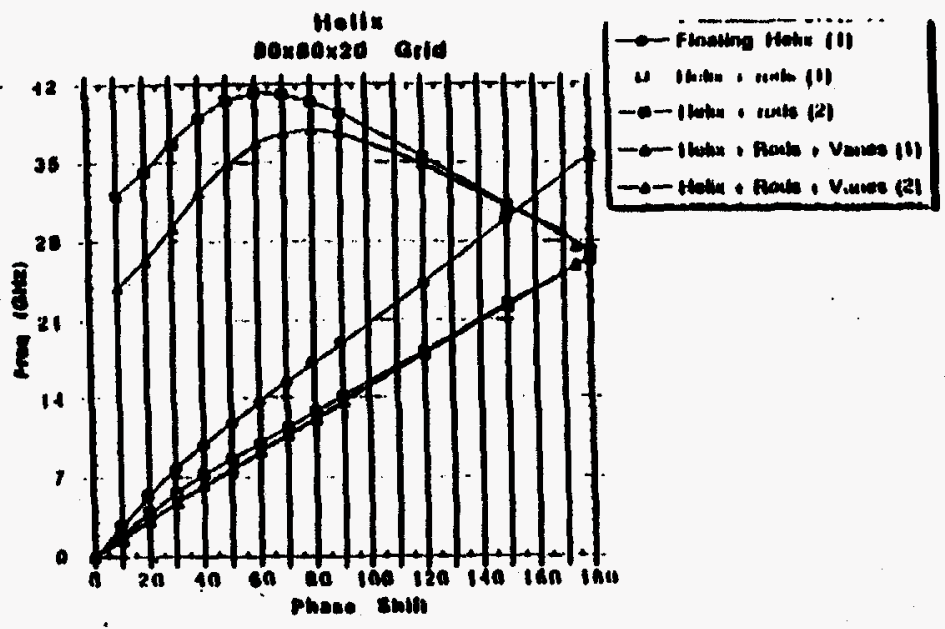

Magire 19. Dhperaica carve for the Nerthrup hellix TWT.

\section{ACKNOWLDDGMENT}

The authors gratefully acknowledge the collaboration of R. Ryne, R.K. Cooper, M.1. Browman, and Q. Rodenz of the LAACG, J. Potter of AccSys Technology. Inc., and $\mathbf{G}$. Dochler of Northrup Corporation in various portions of the material presented in this paper.

- Work supported by the US Department of Bnergy. Division of High Energy Physics under contract number DE-ACOS-91ER40625.

\section{RDTERINACS}

1 A. Meaktofaky, Three-Dimensional Electromagmetic Particle Codes and Applicailons to

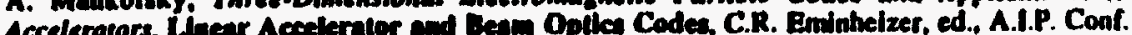
Proc. No. 177 (American Instimule of Puyalcs, New York, 1988), p. I37ff.

2 C.L. Chane, D. Chernila, A. Drobot, K. Ko, M. Kreas, A. Menkofsky, A. Mondelll. and J. Petillo, Three-Dimensional Modeling of Accelerasurs. Proc. of the Conf. on Compulet Codes and the Thes Accelerator Communily, R.K. Cooper, ed. (Los Alamos National Laboratory, January 22 25. 1990. LA-11857-C). p. 27.

3 A. Mankofsky, J.L. Sefior, C.L. Chane, K. Ko, A.A. Mondelli, A.T. Drobot. J. Mourn, W.

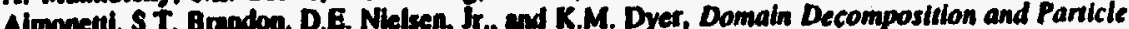
Pushing for Multiprocessing Compulers, Comp. Phys. Commun. 48, 155 (1988). 
- 312 Applicutions of the ARuUS Code

4 E.L. Lindman. "Free-Space" Boundary Conditions for the Time Dependent Wave Equation, 1. Comp. Thys. 18.66 (1975).

$5 \mathrm{~N}$. Kroll and D. Yu, Computer Detenmination of the Exiemal $Q$ and Resonant Frequency of IVavegulde-Lonaded Cavities, Part. Accel. 34, 231 (1991).

6 Y. Goren and D. Yu, Computer-Aided Design of Three-Dimensional Waveguide-Loaded Cavilles. SLAC/AP-73 (1989).

7 R.L. Gluckstem and R. L, Calculation of Caviry Waveguide Coupling, Proc. 1988 Linear Accel. Conf. (Newpon News. VA, Oci. 3-7. 1988. CEBAF Repon 89-131), p. 356. 

support of the U.S. Department of Energy,

Division of High Energy Physics.

\section{COMPUTATIONAL ACCELERATOR PHYSICS LOS ALAMOS, NM 1993 \\ EDITOR: ROBERT RYNE UNIVERSITY OF WISCONSIN}

